\title{
Coastline Identification from Remote-sensing Image Using Informative Vector Machine Learning
}

\author{
Guoshao Su${ }^{1,}$ a, Xiaochuan $\mathrm{Hu}^{1, b^{*}}$, Liubin Yan ${ }^{1, c}$, Yanming $\mathrm{Liu}^{2, \mathrm{~d}}$ \\ ${ }^{1}$ School of Civil Engineering and Architecture, Guangxi University, Nanning, China 530004 \\ ${ }^{2}$ Guangxi College of Water Resources and Electric Power, Nanning, China 530023 \\ asuguoshao@163.com, bh_xchuan@163.com, cyanliubin@126.com, ${ }^{\mathrm{d}}$ YanmingLiu@126.com
}

Keywords: Machine learning, Informative vector machine, Coastline identification.

\begin{abstract}
For a more rapid and accurate coastline identification method, this paper presents a method of coastline identification on remote-sensing image using Informative Vector Machine and then the coastline of Beihai Silver Beach in Guangxi has been identified. The results show that method of coastline identification on remote-sensing using Informative Vector Machine avoids the accuracy deficiency of the edge detection and threshold segmentation, and overcomes a series of open problems that difficulty in determining the optimal network topology structure in artificial neural network and the hyper-parameters in Support Vector Machine. The quick and accurate identification of coastline using the method has been realized which provides an efficient mean for the identification and monitoring of coastline.
\end{abstract}

\section{Introduction}

Coastline is the datum line of distinguishing management area between marine and land. So, quick and accurate measurement and the identification of dynamic change information of coastline are of great importance in Human studies on interaction between sea and land, the effects of the sea activities on the coastal zone, comprehensive protection and utilization offshore resources. The traditional coastline identification methods, which relying on artificial sketch and field measurement, are not able to meet the application of rapid and accurate requirements.

With rapid progress of remote-sensing technology, the coastline identification methods based on remote-sensing image have become an important mean to study space-time evolution of coastal zone area, and a series of achievements have been achieved [1]. For example, Canny operator and the locally adaptive threshold method have been used to extract the coastline, but the identification results are not satisfactory for the complexity of muddy coast edge. The local-threshold segment method has also been used to extract the coastline of remote-sensing image, but the precision of identification results are usually not enough for improper threshold. Contemporary, along with the progress of artificial intelligence discipline, artificial neural network (ANN), Support Vector Machine (SVM) and other intelligence algorithms, combined with remote-sensing technology, have been used for the identification of coastline [2,3].

However, these intelligent algorithms possess a serious of public shortcomings that over-learning and the limitations of poor generalization ability in small sample exist in ANN and hyper-parameters are difficult to choose for SVM. So, looking for a new fast and accurate method of coastline identification based on remote-sensing image is of great importance.

Informative Vector Machine (IVM), a new kind of statistical learning algorithm, put forward by Neil D. Lawrence in 2002, is based on Bayesian approach and kernel-based methods [4, 5]. It gains parameters adaptively and has a strong adaptability to high dimension and complex nonlinear problems, and also is able to make an explanation of the probability. At the same time, the method, based on information entropy theory, selects out part of the most informative points, which constitute the active set, from a large number of training points. Through learning of active set, which can represent the whole training set accurately, as well as a sparse representation for kernel matrix, the computational requirements and the storage requirements of the algorithm are largely 
reduced. Besides, the introduction of assumed density filtering (ADF) and relative information entropy, the method has a strong approximate processing ability to non-Gaussian noise. Therefore, the IVM can be applied to remote-sensing image process and the identification of the coastline.

\section{The Basic Principle of Coastline Identification Using IVM}

The simple greedy selection criterion on which the IVM is based is inspired by information theory. We denote the set of these 'active points' with $I$ and the set of those which are not included with $\boldsymbol{J}$. Initially, $\boldsymbol{I}=\varnothing, \boldsymbol{J}=\{1,2, \ldots, N\}$, and $\boldsymbol{I} \cap \boldsymbol{J}=\varnothing, \boldsymbol{I} \cup \boldsymbol{J}=\{1,2, \ldots, \mathrm{N}\}$ at any time. Also, we assume that the active set, the number is $d$, will be selected from $\mathrm{N}$ training samples. Information vector is achieved in a continuous way which is similar to the online-learning. To begin with, we assume that there are $i$ information vectors, $\boldsymbol{I}_{i}$, and approximate distribution, $q_{\boldsymbol{I}_{i}}(\boldsymbol{f})$, and 1 probability distribution, $p\left(y_{n_{I_{i}}} \mid f_{n_{I_{i}}}\right)$, is as follows:

$$
\left\{\begin{array}{l}
q_{\boldsymbol{I}_{i}}(\boldsymbol{f})=N\left(\boldsymbol{f} ; \boldsymbol{\mu}_{\boldsymbol{I}_{i}}, \boldsymbol{\Sigma}_{\boldsymbol{I}_{i}}\right) \approx p\left(\boldsymbol{f} \mid \boldsymbol{X}_{\boldsymbol{I}_{i},}, \boldsymbol{y}_{\boldsymbol{I}_{i}}, \boldsymbol{\theta}\right) \\
p\left(y_{n_{I_{i}}} \mid f_{n_{I_{i}}}\right) \approx N\left(m_{n_{I_{i}}} ; f_{n_{I_{i}}}, \beta_{n_{I_{i}}}^{-1}\right)
\end{array}\right.
$$

where, the $p$ is probability distribution, $q$ is approximate distribution, $\mu$ is mean value of Gaussian distribution, $\Sigma$ is covariance matrix, $\mathrm{m}$ is likelihood substitution variables, $\beta$ is variance of noise distribution, $\boldsymbol{X}_{\boldsymbol{I}_{i}}$ is input vector of effective training samples, $\boldsymbol{\theta}$ is hyper-parameters of covariance function.

The data-points in $\boldsymbol{I}$ are greedily selected using a simple criterion inspired by information theory: the change in entropy of the posterior process. For an individual point, $n$, as a candidate for the $i$ th inclusion this entropy change is given by:

$$
\Delta H_{I_{i}, j}=-\frac{1}{2} \log \left|\Sigma_{I_{i+1}}\right|+\frac{1}{2} \log \left|\Sigma_{I_{i}}\right|=-\frac{1}{2} \log \left|\Sigma_{I_{i+1}} \Sigma_{I_{i}}^{-1}\right|
$$

This entropy change can be evaluated for every point in the inactive set, $J$, and the point associated with the largest entropy decrease can then be included.

This process is repeated until $d$ inclusions have been made so that the learning of active set, $\boldsymbol{I}$, can be achieved instead of the original sample data set. And then:

$$
\left\{\begin{array}{l}
p\left(\boldsymbol{y}_{\boldsymbol{I}} \mid \boldsymbol{X}_{\boldsymbol{I},:}, \boldsymbol{\theta}\right) \approx N\left(\boldsymbol{m}_{\boldsymbol{I}} ; \mathbf{0}, \boldsymbol{K}_{\boldsymbol{I}}+\boldsymbol{B}_{I}^{-1}\right) \\
p\left(\boldsymbol{f} \mid \boldsymbol{y}_{\boldsymbol{I}}, \boldsymbol{X}_{\boldsymbol{I},:}, \boldsymbol{\theta}\right) \approx N\left(\boldsymbol{f} ; \boldsymbol{\mu}_{I}, \Sigma_{I}\right) \\
\boldsymbol{\mu}_{\boldsymbol{I}}=\Sigma_{\boldsymbol{I}} \boldsymbol{B}_{I} \boldsymbol{y}_{\boldsymbol{I}}, \Sigma_{\boldsymbol{I}}=\left(\boldsymbol{B}_{\boldsymbol{I}}+\boldsymbol{K}_{I}^{-1}\right)^{-1}
\end{array}\right.
$$

where, $\boldsymbol{B}$ is variance of noise distribution, $\boldsymbol{K}$ or $\Sigma$ is covariance matrix of Gaussian distribution, and each element is covariance of any two input vectors.

Based on the Eq. (3), we can achieve the posterior distribution of IVM:

$$
\left\{\begin{array}{l}
p\left(f_{*} \mid \boldsymbol{y}, \boldsymbol{X}, \boldsymbol{x}_{*}, \boldsymbol{\theta}_{l}\right) \approx p\left(f_{*} \mid \boldsymbol{y}_{\boldsymbol{I}}, \boldsymbol{X}_{I,:}, \boldsymbol{x}_{*}, \boldsymbol{\theta}_{l_{I}}\right)=N\left(f_{*} ; \mu_{*}, \sigma_{*}^{2}\right) \\
\mu_{*}=\boldsymbol{K}_{* I}^{\mathrm{T}} \boldsymbol{K}_{\boldsymbol{I}}^{-1} \Sigma_{\boldsymbol{I}} \boldsymbol{B}_{I} \boldsymbol{y}_{\boldsymbol{I}}, \sigma_{*}^{2}=k_{*}+\boldsymbol{K}_{* \boldsymbol{I}}^{\mathrm{T}} \boldsymbol{K}_{\boldsymbol{I}}^{-1}\left(\Sigma_{\boldsymbol{I}}-\boldsymbol{K}_{\boldsymbol{I}}\right) \boldsymbol{K}_{\boldsymbol{I}}^{-1} \boldsymbol{K}_{* \boldsymbol{I}}
\end{array}\right.
$$

where, $\boldsymbol{X}$ is input vector of the training sample points and $\boldsymbol{x}^{*}$ is input vector of forecast samples to be inputted. Based on the Eq. (4), we can achieve IVM classifier with generalization ability. The IVM classifier is:

$$
\left\{\begin{array}{l}
p\left(y_{*}=+1 \mid \boldsymbol{X}, \boldsymbol{y}, \boldsymbol{x}_{*}\right)=\int \Phi\left(f_{*}\right) p\left(f_{*} \mid \boldsymbol{y}, \boldsymbol{X}, \boldsymbol{x}_{*}, \boldsymbol{\theta}_{l}\right) d f_{*}=\Phi\left(\frac{\mu_{*}}{\sqrt{1+\sigma_{*}^{2}}}\right) \\
\text { if } p \geq 0.5, \quad y_{*}=+1, \text { otherwise, } y_{*}=-1
\end{array}\right.
$$


where, the $\Phi(x)$ is cumulative probability density function of standard normal distribution.

Inputting the input vector of forecast samples, $\boldsymbol{X}_{*}$, into IVM classifier, we can achieve the corresponding forecast output target, $\boldsymbol{y}_{* .}$ And then, when the forecast output target of pixel, $\boldsymbol{y}_{*}$, is "-1", the pixel will be classified as the water; when $\boldsymbol{y}_{*}$ is " +1 ", the pixel will be classified as land. Until all pixels of remote-sensing image are classified, the segmentation of remote-sensing image is finished. Based on the completed segmentation of remote sensing image, gray processing and binarization can be conducted in MATLAB. At last, the coastline can be achieved based on the binary image.

\section{Application Example}

TM image, sensor is TM and orbital is 125/045, in coastal waters of Beihai Silver Beach in Guangxi was used for band combination, geometric correction, clipping and other pretreatments. TM image was got on November 17, 2001 and its spatial resolution is thirty meters. It is worth mentioning that the fourth band, near infrared band, and the third band, red band, and the second band, green band were used for band combination. And then, standard false color image for coastline identification was obtained. At last, remote sensing image that contains coastline information was acquired. The remote-sensing image shows in Fig. 1.

\section{The Process of Coastline Identification Using IVM}

Step 1. Calculating normalized difference water index (NDWI) is necessary so that initial distinguish between water and land can be realized. Then, the results of calculating, Fig. 2, can be achieved.

$$
N D W I=\left(\rho_{\text {Green }}-\rho_{\text {NIR }}\right) /\left(\rho_{\text {Green }}+\rho_{\text {NIR }}\right)
$$

where, the $\rho_{\text {Green }}$ is green band and the $\rho_{N I R}$ is near-infrared band.

Step 2. Basing on the result of calculating, NDWI, 100 sample points of water and 100 sample points of land will be selected in remote-sensing image. It is worth mentioning that the sample points must be representative for water and land.

Step 3. Texture characteristic value of sample points will be extracted and it will compose the input vector, $\boldsymbol{x}_{j=}\left[\mathrm{asm}_{j}, \mathrm{con}_{j}, \mathrm{idm}_{j}\right.$, ent $]$ ], of the training samples. Where asm is energy, con is contrast, idm is relevance, ent is entropy, and all of these values of $\boldsymbol{x}_{j}$ are extracted in Gray symbiotic matrix which is based on the results of NDWI. The training sample is made up of $\boldsymbol{x}_{j}$ and $\boldsymbol{y}_{j}$ and $\boldsymbol{y}_{j}$ is output observations. As the sample points are water, $\boldsymbol{y}_{j}$ is ' -1 ', otherwise ' +1 '. Where training sample is $\left(\boldsymbol{x}_{j}, \boldsymbol{y}_{j}\right), 1 \leq j \leq 200$, and $j$ is the number of sample set.

Step 4. Texture characteristic value of all pixel in remote-sensing image will be extracted and it compose the input vector of the test samples, $\boldsymbol{X}_{*}, \boldsymbol{X}_{*}=\left[\boldsymbol{x}_{1}, \boldsymbol{x}_{2}, \ldots \boldsymbol{x}_{M}\right]^{\mathrm{T}}$, and $M$ is number of all pixel of remote-sensing image.

Step 5. Inputting the training samples, $\left(\boldsymbol{x}_{j}, \boldsymbol{y}_{j}\right)$, into the IVM, and then the classifier, Eq. (5) will be obtained.

Step 6. The output value, $y^{*}$, can be obtained by inputting the $\boldsymbol{X}_{*}$ into classifier, Eq. (5). And then, the segmentation of remote sensing image has been finished and the result is shown in Fig. 3 . As $y_{*}=-1$, the pixel is classified as waters, otherwise land.

Step 7. Sensing segmentation results, shown in Fig. 3, classified in above steps is translated into Binary image, Fig. 5, after graying and binaryzation, according to grey histogram, Fig. 4.

Step 8. The accurate coastline, shown in Fig. 6, is obtained by means of vectorization which is based on Fig. 5. 


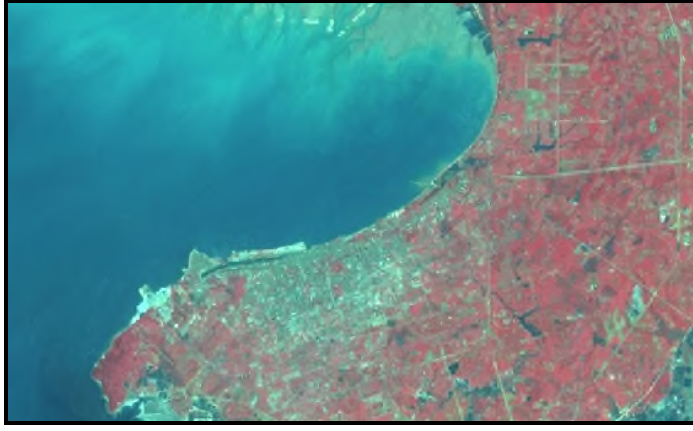

Fig. 1 Remote sensing image.

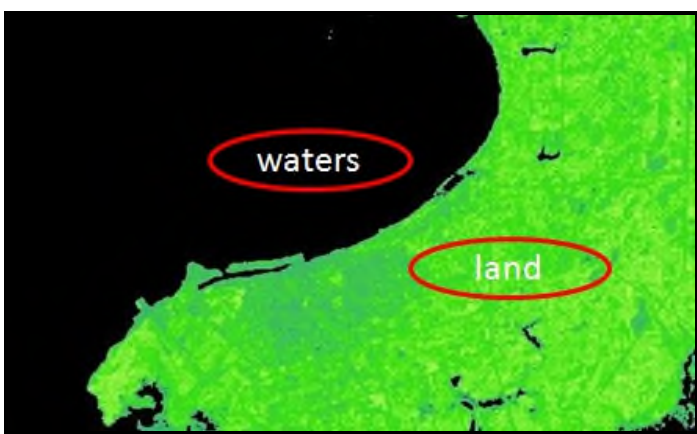

Fig. 3 Sensing segmentation results.

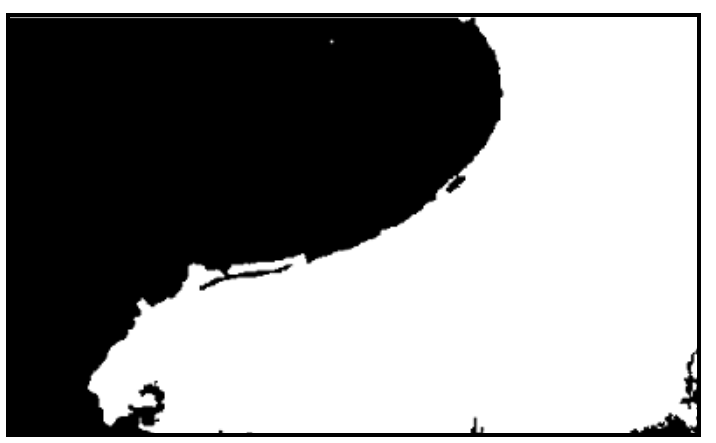

Fig. 5 The binary image.

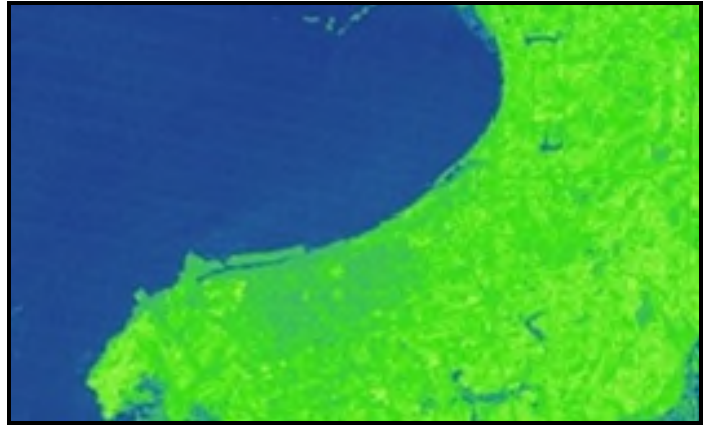

Fig. 2 The result of NDWI.

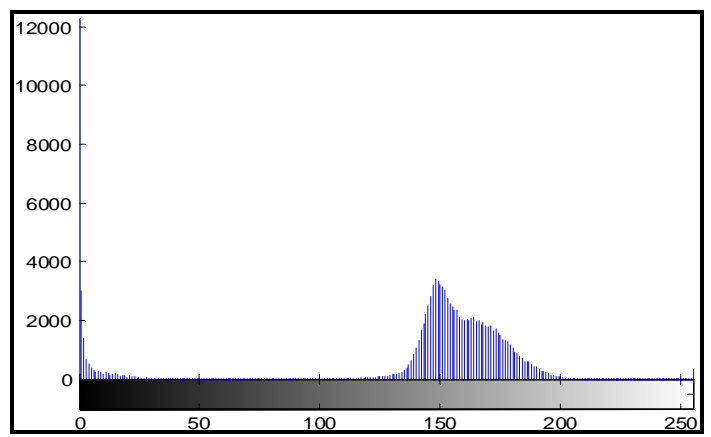

Fig. 4 The grey histogram.

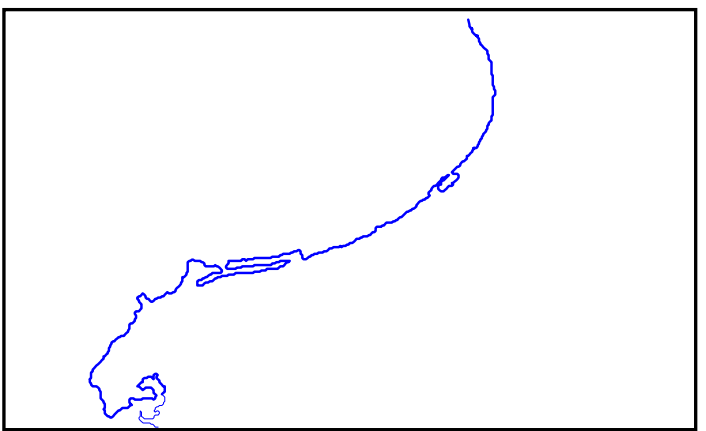

Fig. 6 The result of coastline identification.

\section{The Results Analysis of Coastline Identification}

Texture, reflecting the interrelation between object and the neighborhood, is a kind of local spatial information and also overcome the defect of spectral signature which does not take into account the space information. It is for this reason, the textural features is able to reduce the noise effect on the regional characteristics. So, training samples composed by textural features are helpful to the identification of coastline.

Fig. 3 is the segmentation results using IVM. Waters and land can be identified effectively through learning and training of samples. Also, we found that IVM is less time consuming than SVM which means it is a more efficient way to extracting the coastline. Based on Fig. 3, coastline can be obtained by graying, binaryzation and vectorization.After graying of Fig. 3, the binaryzation, the threshold value is 60, has been achieved according to grey histogram, Fig. 4. At last, we can get the coastline by vectorization on Fig. 5 .

Fig. 6, the outcome of vectorization, is the coastline of Beihai Silver Beach in Guangxi. By comparison between Fig. 6 and Fig. 1, the coastline goes with Silver Beach well. So the method using IVM is feasible in practical engineering. 


\section{Conclusions}

In this paper, IVM has been used for coastline Identification of Beihai Silver Beach in Guangxi which is based on training and learning of textural features on remote-sensing image. The results show that the method avoids the accuracy deficiency of the edge detection and threshold segmentation, and overcomes a series of open problems in ANN and SVM. Meanwhile, the method provides an efficient way for the identification and monitoring of coastline which also provides a good application value in engineering for the protection of the coastal landform and the orderly development of coastal resources.

\section{Acknowledgements}

This work was financially supported by the science-technology projects in the water resources department of Guangxi province, China (Grant No. 201522)

\section{References}

[1] H. G. Sohn, K. C. Jezek. Mapping ice sheet margins from ERS-1 SAR and SPOT imagery J. Int. J. Remote Sens. 20(15-16) (1999) 3201-3216.

[2] T. W. Ryan, P. J. Sementilli, P. Yuen. Extraction of shoreline features by neural nets and image processing J. Photogram. Eng. Remote Sens. 57(7) (1991) 947-955.

[3] C. M. Zhu, X. Zhang, J. C. Luo, et al. Automatic extraction of coastline by remote sensing technology based on SVM and auto-selection of training samples. Remote Sens. Land Res. 25(2) (2013) 69-74.

[4] D. L. Neil, C. P. John, Learning to learn with the informative vector machine, ACM, 2004.

[5] L. Neil, S. Matthias, H. Ralf, Fast sparse Gaussian process methods: The informative vector machine, (2003), pp. 609-616. 